\title{
USO DA ESPECTROFOTOMETRIA UV-VIS PARA DIFERENCIAR AS COLORAÇÕES DO BIODIESEL DE PALMA E DO CORANTE MARCADOR DE ÓLEO DIESEL
}

\author{
Maria S. M. Quintino, ${ }^{\mathrm{a}, *}$, Waldemar P. Oliveira Filho ${ }^{\mathrm{a}}$, Fábio S. Vinhado ${ }^{\mathrm{a}}$, Warley G. Santos ${ }^{\mathrm{a}}$, Rodrigo B. C. Dutra ${ }^{\mathrm{b}}$, Patrícia \\ A. Oliveira ${ }^{b}$, Patrícia K. G. Costa ${ }^{b}$ e Itânia P. Soares ${ }^{b}$ \\ ${ }^{a}$ Centro de Pesquisas e Análises Tecnológicas, Agência Nacional do Petróleo, Gás Natural e Biocombustíveis, 70830-902, Brasília \\ - DF, Brasil

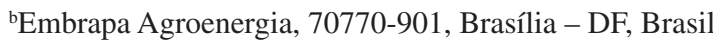

Recebido em 15/09/2016; aceito em 01/02/2017; publicado na web em 05/04/2017

\begin{abstract}
RAPID SPECTROPHOTOMETRIC DIFFERENTIATION BETWEEN PALM OIL BIODIESEL AND RED DYE ADDED TO DIESEL FUEL. Currently, there are two mixtures of biodiesel/diesel fuel sold in Brazil: BX/S500 and BX /S10 (X = volume/volume biodiesel percentage; S500 and S10 = sulfur content in diesel fuels - 500 ppm and 10 ppm, respectively). In order to differentiate these mixtures, red dye is added to diesel oil as required by the National Agency for Petroleum, Natural Gas and Biofuels (ANP), regulatory agency in Brazil. Since the palm oil biodiesel has also a reddish color, mixing this product with diesel fuel leads to difficulty in visual differentiation of the mixtures BX/S500 and BX/S10. We propose the use of UV-Vis spectrophotometry, which is a low cost, simple and rapid technique. Spectra obtained for different mixtures show that qualitative distinction is possible. For quantification of red dye in the presence of palm oil biodiesel, the method is limited when dye is present in low concentrations $\left(<0.1 \mathrm{mg} \mathrm{L}^{-1}\right)$ due to matrix effects. The method showed good repeatability of the absorbance responses, wide linear dynamic range $\left(0.02 \mathrm{mg} \mathrm{L}^{-1}\right.$ to $\left.40.00 \mathrm{mg} \mathrm{L}^{-1}\right)$ and low limits of detection and quantification $\left(0.006 \mathrm{mg} \mathrm{L}^{-1}\right.$ and $0.019 \mathrm{mg} \mathrm{L}^{-1}$, respectively).
\end{abstract}

Keywords: palm oil biodiesel; diesel fuel; red dye; spectrophotometry.

\section{INTRODUÇÃO}

Atualmente, o óleo de soja e o sebo bovino são as duas fontes de matéria-prima mais utilizadas para a produção de biodiesel no Brasil, respondendo por mais de $90 \%$ de todo o biocombustível fabricado no país. Dentre outras matérias-primas que possuem uma participação menor, pode-se citar o dendê. ${ }^{1}$

O óleo de dendê (ou óleo de palma), que representa um terço do óleo vegetal comercializado no mundo e possui produtividade dez vezes maior do que o de soja, poderá experimentar uma produção crescente no Brasil. O projeto de lei da Câmara dos Deputados PLC 119/2013, aprovado pela Comissão de Meio Ambiente, Defesa do Consumidor e Fiscalização e Controle (CMA) do Senado Federal, ${ }^{2}$ criou o Programa de Produção Sustentável da Palma de Óleo no Brasil, em que ficam estabelecidas diretrizes para proteção do meio ambiente, conservação da biodiversidade, utilização racional dos recursos naturais e respeito à função social da propriedade. O programa prevê incentivos para o plantio da palmeira que produz o dendê, nativa ou exótica, inclusive para recomposição da reserva florestal em propriedades localizadas na Amazônia Legal. ${ }^{2}$

Hoje, no país, a participação dessa matéria-prima na obtenção do biodiesel é pouco significativa, uma vez que a produção de óleo ainda é baixa. Ainda assim, a planta pode ganhar maior participação entre as fontes de óleo para a produção do biocombustível. ${ }^{3}$ Atualmente, o Brasil produz em torno de 420 t/ano, enquanto a produção mundial é próxima de 60 milhões t/ano. ${ }^{4}$

No Brasil, o biodiesel é usado como combustível em misturas com óleo diesel e deve atender as especificações da ANP. ${ }^{5}$ Para identificar a concentração do biodiesel na mistura, passou-se a adotar uma nomenclatura bastante apropriada: biodiesel $\mathrm{BX}$, onde $\mathrm{X}$ é a percentagem, em volume, do biodiesel na mistura. Por exemplo, B5,

*e-mail: mquintino@anp.gov.br
B7, B20 e B100 são combustíveis com uma concentração de 5\%,7\%, $20 \%$ e $100 \%$ (v/v) de biodiesel, respectivamente.

Atualmente, existem duas misturas de óleo diesel/biodiesel comercializadas no país: o BX com S500 e o BX com S10, contendo 500 ppm e 10 ppm de enxofre, respectivamente. Para identificar o tipo de BX comercializado nos postos de combustível, a ANP determina que o óleo diesel A S500 seja marcado com um corante vermelho. A especificação para a mistura BX é regulamentada pela Resolução $\operatorname{ANP}^{\circ}$ 50/2013. ${ }^{6}$

Sabe-se que, em razão da presença de carotenoides, o óleo de palma apresenta coloração avermelhada. Sendo assim, a mistura de biodiesel de palma com o óleo diesel S10 pode resultar em um produto considerado fora da especificação, já que este deve apresentar coloração amarela.

A presente proposta de estudo surge como resultado de uma necessidade do mercado nacional de combustíveis, a qual consiste em diferenciar o óleo diesel B S10 e o óleo diesel B S500, quando o primeiro ou ambos venham conter biodiesel de óleo de palma. A diferença óbvia entre esses produtos, em uma primeira análise, está no teor de enxofre, o qual poderia ser quantificado por meio do uso de métodos estabelecidos e, inclusive, especificados pela ANP, como por exemplo: Fluorescência no Ultravioleta, Espectrometria de Emissão Óptica com Plasma Indutivamente Acoplado (ICP-OES) e Espectrometria de Fluorescência de Raios X (Energia Dispersiva). Entretanto, a principal motivação da proposta está relacionada ao desejo de efetuar essa distinção por meio do emprego de um método simples, de baixo custo e que ofereça tempo de análise reduzido, em comparação com os métodos apresentados para a determinação do teor de enxofre e previstos na Resolução ANP n ${ }^{\circ}$ 30/2016. ${ }^{6}$

Uma metodologia simples que, em princípio, poderia ser empregada para distinguir esses produtos envolve o uso da técnica Espectrofotometria de Absorção Molecular na Região do UltravioletaVisível (UV-Vis). De acordo com Corgozinho, ${ }^{7}$ o corante utilizado para marcar o óleo diesel apresenta duas bandas de absorção, uma 
em $380 \mathrm{~nm}$ e outra em $540 \mathrm{~nm}$. No referido estudo, a banda correspondente à absorção em $540 \mathrm{~nm}$ não foi observada para a amostra diluída de óleo diesel marcado com o corante. No entanto, a banda se apresenta quando a amostra sem diluição é empregada. Remédios e colaboradores ${ }^{8}$ obtiveram espectros de absorbância de diferentes óleos derivados de frutos da palma e observaram que todos apresentaram uma única banda de absorção dentro do intervalo de 250 a $600 \mathrm{~nm}$. Dal Prá e colaboradores ${ }^{4}$ e Szydłowska-Czerniak e colaboradores ${ }^{9}$ quantificaram os carotenoides presentes no óleo de palma (Elaeis guineenis) pela absorbância a $450 \mathrm{~nm}$. Considerando que o óleo de palma possui potencial para produção de biodiesel, é importante o desenvolvimento de uma metodologia que permita distinguir, de forma simples, rápida e inequívoca, a coloração da mistura biodiesel de palma/óleo diesel e das misturas biodiesel/óleo diesel e biodiesel de palma/óleo diesel contendo corante vermelho. O presente trabalho se propôs a buscar diferenciar os óleos diesel B S10 e S500 (contendo biodiesel de óleo de palma), levando em consideração a absorção dos produtos em regiões do UV-Visível. Inicialmente, foi realizado um estudo qualitativo para confirmar a possibilidade de diferenciar os referidos produtos. Em etapa posterior foram realizados estudos complementares para a quantificação do corante Sudan M Vermelho em diferentes amostras de óleo diesel contendo biodiesel de palma.

\section{PARTE EXPERIMENTAL}

\section{Aparelhagem, reagentes, soluções e amostras}

Amostras

O óleo de palma (Elaeis guineensis) bruto foi doado pela empresa JBS S.A., em São Paulo.

\section{Síntese da amostra de biodiesel de palma}

A amostra de biodiesel foi sintetizada em reator de vidro Büchi, mini pilot 5. Para tanto, foram utilizados: óleo de palma (1000 g), metanol anidro (Sigma Aldrich), hidróxido de potássio (Synth) e ácido sulfúrico (J.T. Baker). Inicialmente, foi feita uma reação de esterificação empregando uma razão molar 8:1 de metanol/óleo e $1 \%$ de ácido sulfúrico como catalisador, a uma temperatura de 60 ${ }^{\circ} \mathrm{C}$, durante 1 hora. Em seguida, a amostra foi submetida ao processo de secagem para retirada do excesso de metanol e água. Após essa etapa, foi feita a reação de transesterificação a $60^{\circ} \mathrm{C}$ durante 1 hora, com razão molar 6:1 de metanol/óleo e 1\% de $\mathrm{KOH}$ (catalisador).

Caracterização físico-química da amostra de biodiesel de palma

A amostra de biodiesel de palma obtida conforme descrição acima foi submetida à caracterização quanto ao atendimento da especificação constante da Resolução ANP no 45/2014. ${ }^{5}$ Para tanto, foram empregados os seguintes equipamentos (Tabela 1):

Tabela 1. Equipamentos empregados na caracterização da amostra de biodiesel de palma

\begin{tabular}{lc}
\hline Característica & Equipamentos/Aparelhagem \\
\hline Massa Específica a $20^{\circ} \mathrm{C}$ & Rudolph Research Analytical/DDM \\
2911 \\
\hline Viscosidade Cinemática a $40^{\circ} \mathrm{C}$ & Herzog/HVM 472 \\
\hline Teor de água & Metrohm/titrando 907 \\
\hline Ponto de Fulgor & ISL/FP93 5G2 \\
\hline Enxofre Total, Na + K, Ca $+\mathrm{Mg}, \mathrm{P}$ & ICP PerkinElmer/ Optima 8300 \\
\hline Estabilidade à Oxidação a $110^{\circ} \mathrm{C}$ & Metrohm/Rancimat 873 \\
\hline $\begin{array}{l}\text { Teor de éster, Metanol, Glicerina Livre } \\
\text { e Total; Mono-, Di- e Triglicerídeos }\end{array}$ & GC/FID Agilent/7890 A \\
\hline
\end{tabular}

Diferenciação espectrofotométrica e quantificação do corante

Para os ensaios envolvendo a diferenciação das colorações atribuídas ao biodiesel de palma e ao corante Sudan M Vermelho, foram utilizados um Espectrofotômetro Cary $60 \mathrm{UV}$-Vis, Agilent Technologies, com interface ao software Cary Win UV, célula de quartzo de $1 \mathrm{~cm}$ de caminho óptico e varredura de $60 \mathrm{~nm} \mathrm{~min}^{-1}$.

A Tabela 2 relaciona os principais padrões e reagentes empregados nesta etapa do estudo.

Tabela 2. Relação dos principais padrões/reagentes empregados nas etapas de diferenciação espectrofotométrica e quantificação do corante

\begin{tabular}{|c|c|c|}
\hline Item & Material & Procedência \\
\hline 1 & Óleo diesel S500 A & REPLAN \\
\hline 2 & Óleo diesel S10 A & REVAP \\
\hline 3 & B100 de soja & Amostra PIB \\
\hline 4 & B100 de palma & Amostra sintetizada em laboratório \\
\hline 5 & \multicolumn{2}{|c|}{$\begin{array}{l}\text { Corante Sudan M Verme-BASF } \\
\text { lho } 462\end{array}$} \\
\hline 6 & Tolueno & Impex $(99,5 \%$ de pureza) \\
\hline 7 & Hexano & J. T. Baker $(99,8 \%$ de pureza) \\
\hline 8 & S500 B7 de palma & $\begin{array}{l}\text { Amostra preparada utilizando } 7 \% \mathrm{v} / \mathrm{v} \\
\text { do item } 4 \text { no item } 1\end{array}$ \\
\hline 9 & S10 B7 de soja & $\begin{array}{l}\text { Amostra preparada utilizando } 7 \% \mathrm{v} / \mathrm{v} \\
\text { do item } 3 \text { no item } 2\end{array}$ \\
\hline
\end{tabular}

REPLAN = Refinaria de Paulínia (Petrobras); REVAP = Refinaria Henrique Lage (Petrobras); PIB = Programa de Comparação Interlaboratorial em Biodiesel (ANP).

\section{Preparação de soluções e amostras}

As soluções-padrão utilizadas para a obtenção das curvas analíticas foram preparadas mediante diluições a partir de uma solução-estoque do corante Sudan M Vermelho, empregando tolueno como solvente de diluição.

Amostras analisadas quanto à quantificação do corante em presença da palma foram preparadas em laboratório, conforme descrição constante na Tabela 3.

As amostras empregadas no estudo qualitativo (ensaios envolvendo espectrofotometria de varredura) são identificadas ao longo da seção Discussão dos Resultados.

Tolueno, hexano e os óleos diesel A S500 e S10 foram empregados como soluções-branco, conforme a necessidade, para zerar o espectrofotômetro.

\section{RESULTADOS E DISCUSSÃO}

\section{Caracterização físico-química da amostra de biodiesel de palma}

Os resultados para a caracterização da amostra de biodiesel de palma são apresentados na Tabela 4.

De maneira geral, os parâmetros obtidos para a amostra de biodiesel se mantiveram dentro da especificação prevista pelo Regulamento Técnico ANP no 03/2014, constante da Resolução ANP n ${ }^{\circ} 45 / 2014 .{ }^{5}$ As características Teor de Água e Diglicerídeos também se encontram de acordo com a especificação, considerando uma tolerância calculada, conforme estabelecido pela norma ISO 4259, que usa 0,59 x R, em que $\mathrm{R}$ é a reprodutibilidade da norma (Tabela 5). Ponto de Fulgor e Metanol apresentaram resultados fora da especificação, que indicam que o reagente metanol usado para 
Tabela 3. Descrição das amostras utilizadas na quantificação do corante Sudan M Vermelho

\begin{tabular}{|c|c|c|}
\hline Amostra & $\begin{array}{l}\text { Concentração nominal } \\
\text { do corante na amostra }\end{array}$ & Descrição do preparo \\
\hline 1 & $0,372 \mathrm{mg} \mathrm{L}^{-1}$ & $\begin{array}{l}500 \mu \mathrm{L} \text { de } \mathrm{S} 500 \mathrm{~B} 7 \text { de palma contendo } \\
\text { corante Sudan M Vermelho } 18,6 \mathrm{mg} \mathrm{\textrm {L } ^ { - 1 }} \\
\text { foram transferidos para balão de } 25 \mathrm{~mL} \text {, } \\
\text { avolumando-se com S10 B7 de soja. }\end{array}$ \\
\hline 2 & $7,020 \mathrm{mg} \mathrm{L}^{-1}$ & $\begin{array}{l}1,95 \mathrm{~mL} \text { de uma solução de corante } \\
\text { Sudan } \mathrm{M} \text { Vermelho } 90,0 \mathrm{mg} \mathrm{L}^{-1}+1,75 \\
\mathrm{~mL} \text { de B100 de palma foram transferidos } \\
\text { para balão de } 25 \mathrm{~mL} \text {, avolumando-se } \\
\text { com óleo diesel S500 A REPLAN. }\end{array}$ \\
\hline 3 & $18,612 \mathrm{mg} \mathrm{L}^{-1}$ & $\begin{array}{l}5,17 \mathrm{~mL} \text { de uma solução de corante } \\
\text { Sudan M Vermelho } 90,0 \mathrm{mg} \mathrm{L}^{-1}+1,75 \\
\mathrm{~mL} \text { de B100 de palma foram transferidos } \\
\text { para balão de } 25 \mathrm{~mL} \text {, avolumando-se } \\
\text { com óleo diesel S500 A REPLAN. }\end{array}$ \\
\hline
\end{tabular}

Tabela 4. Resultado dos ensaios de caracterização da amostra de biodiesel de palma

\begin{tabular}{lcc}
\hline Característica & Resultados & Método \\
\hline Massa Específica a $20^{\circ} \mathrm{C}$ & $873,4 \mathrm{~kg} \mathrm{~m}^{-3}$ & ASTM D4052 \\
Viscosidade Cinemática a $40^{\circ} \mathrm{C}$ & $4,43 \mathrm{~mm}^{2} \mathrm{~s}^{-1}$ & ASTM D445 \\
Teor de água & $354,6 \mathrm{mg} \mathrm{kg}^{-1}$ & ASTM D6304 \\
Ponto de Fulgor & $79{ }^{\circ} \mathrm{C}$ & ASTM D93 \\
Enxofre & $<10 \mathrm{mg} \mathrm{kg}^{-1}$ & NBR 15867 \\
$\mathrm{Na}+\mathrm{K}$ & $<\mathrm{LD}$ & EN14538:2006 \\
$\mathrm{Ca}+\mathrm{Mg}$ & $0,6 \mathrm{mg} \mathrm{kg}^{-1}$ & EN14538:2006 \\
$\mathrm{P}$ & $<5 \mathrm{mg} \mathrm{kg}^{-1}$ & NBR 15553 \\
Estabilidade à Oxidação a $110^{\circ} \mathrm{C}$ & $>30 \mathrm{~h}^{-1}$ & EN14112 \\
Teor de éster & $99,665 \%\left(\mathrm{~m} \mathrm{~m}^{-1}\right)$ & EN 14103:2011 \\
Glicerina Livre & $0,003 \%\left(\mathrm{~m} \mathrm{~m}^{-1}\right)$ & ASTM D6584 \\
Monoglicerídeos & $0,425 \%\left(\mathrm{~m} \mathrm{~m}^{-1}\right)$ & ASTM D6584 \\
Diglicerídeos & $0,223 \%\left(\mathrm{~m} \mathrm{~m}^{-1}\right)$ & ASTM D6584 \\
Triglicerídeos & $0,113 \%\left(\mathrm{~m} \mathrm{~m}^{-1}\right)$ & ASTM D6584 \\
Glicerina Total & $0,158 \%\left(\mathrm{~m} \mathrm{~m}^{-1}\right)$ & ASTM D6584 \\
Metanol & $0,28 \%\left(\mathrm{~m} \mathrm{~m}^{-1}\right)$ & EN 14110 \\
& & (procedimento B) \\
\hline
\end{tabular}

Tabela 5. Tolerâncias calculadas a partir das reprodutibilidades das normas para os parâmetros Teor de Água e Diglicerídeos, conforme determinado pela ISO 4259

\begin{tabular}{lcc}
\hline & Teor de Água & Diglicerídeos \\
\hline Norma utilizada & ASTM D6304 & ASTM D6584 \\
Reprodutibilidade (R) & $\mathrm{R}=0,4243^{*} \mathrm{X}^{0,6}$ & $\mathrm{R}=0,784 * \mathrm{X}^{0,93}$ \\
X (limite na especificação $\left.{ }^{5}\right)$ & $350 \mathrm{ppm}$ & $0,20 \%\left(\mathrm{~m} \mathrm{~m}^{-1}\right)$ \\
Reprodutibilidade calculada & 14,3 & 0,18 \\
Tolerância calculada & 358 & 0,30 \\
\hline
\end{tabular}

a transesterificação não foi completamente lavado, o que não deve influenciar nas características de cor.

Embora não tenha sido possível obter todos os parâmetros determinados na Resolução ANP 45/20145 (ou ainda, todos dentro da especificação), faz-se importante ressaltar que tal fato não deve exercer influência sobre o objetivo do estudo, uma vez que este se baseia na diferenciação espectrofotométrica dos principais compostos coloridos que compõem as amostras, não tendo relação com os demais ensaios previstos.

\section{Palma vs. Corante: diferenciação espectrofotométrica qualitativa}

Com vistas a conhecer o comportamento das espécies consideradas neste estudo, foram realizados ensaios espectrofotométricos de varredura, considerando soluções diluídas e outras que não foram submetidas a diluições.

Na Figura 1 é apresentado o espectro de absorção obtido para uma solução $20 \mathrm{mg} \mathrm{L}^{-1}$, em hexano, do corante Sudan M Vermelho. O hexano foi usado porque possui uma absorbância desprezível na região do ultravioleta acima de um comprimento de onda de 200 nm. O espectro (Figura 1) apresenta duas bandas de absorção com máximos em $372 \mathrm{~nm}$ e $527 \mathrm{~nm}$, os quais se situam próximo àqueles reportados na literatura. ${ }^{7}$ Além disso, esses resultados estão de acordo com os requisitos exigidos pela Resolução ANP no 30/2016 ${ }^{6}$ para a especificação do corante vermelho para o óleo diesel S500.

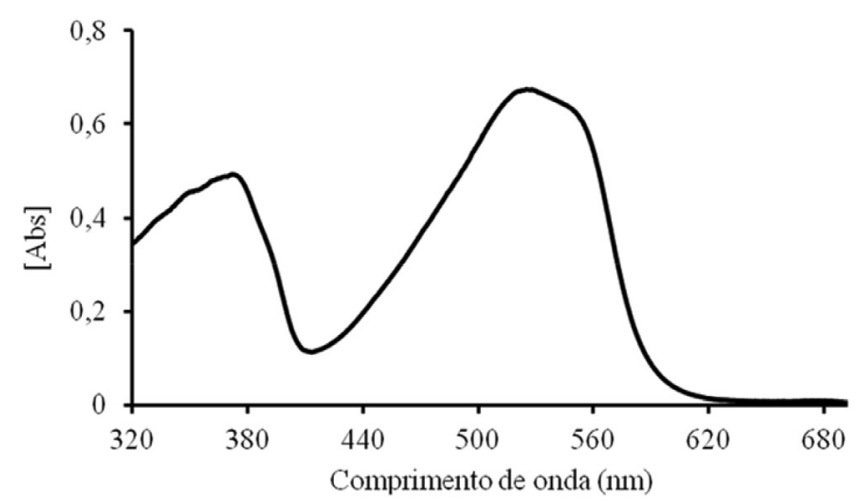

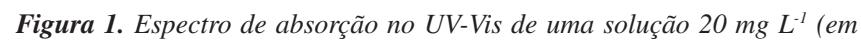
hexano) do corante Sudan M Vermelho

A Figura 2 traz os espectros UV-Vis de soluções de biodiesel de óleo de palma e de biodiesel de soja, que foram previamente submetidas à diluição com hexano (270 vezes). O biodiesel de soja, cujo espectro é similar àquele obtido para hexano, não apresenta bandas de absorção. Já para o biodiesel de palma pode ser observada uma banda com absorbância máxima em comprimento de onda de 444 $\mathrm{nm}$, aproximadamente, característica das ligações duplas conjugadas dos compostos $\beta$-caroteno, ${ }^{10}$ presentes no óleo de palma.

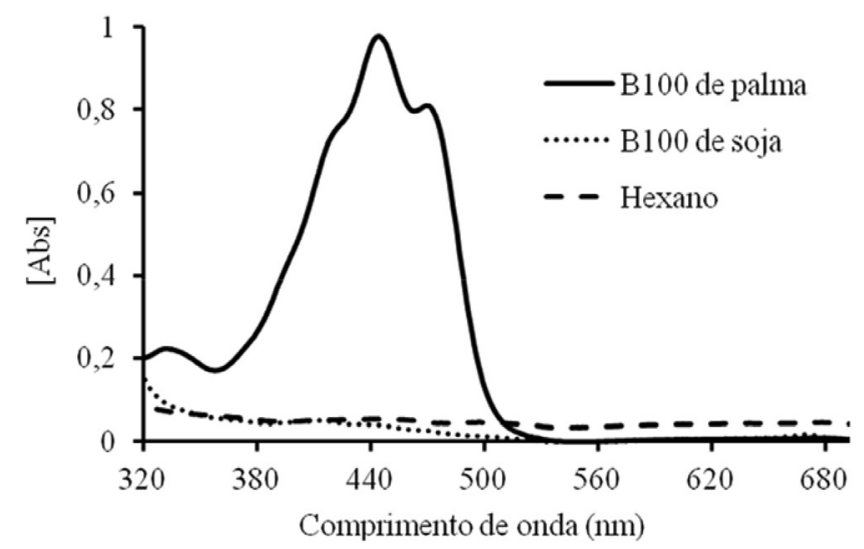

Figura 2. Espectros de absorção no UV-Vis obtidos para hexano e para soluções diluídas de B100 de palma e de B100 de soja (diluídas 270 vezes com hexano) 
Na Figura 3 pode ser observada a sobreposição dos espectros de diferentes soluções, no intervalo compreendido entre 380-700 nm. As soluções não foram submetidas às diluições.

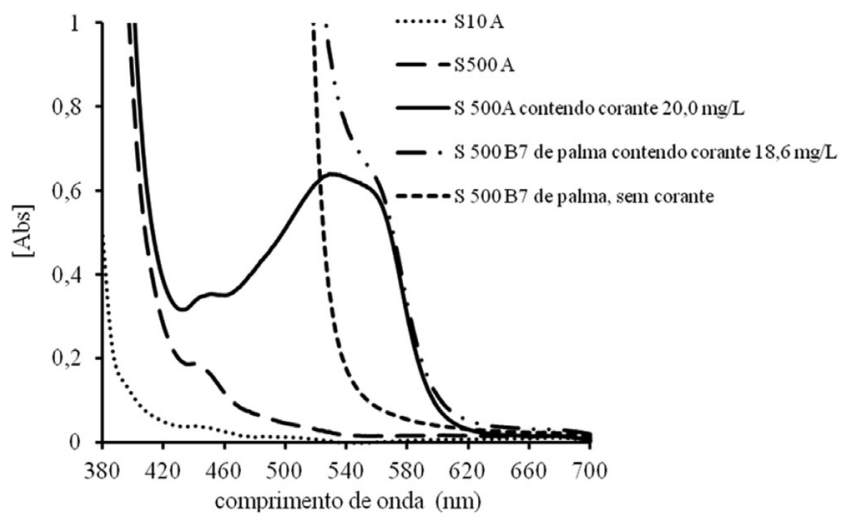

Figura 3. Espectros de absorção obtidos para os óleos diesel A S10 e S500 e para as soluções $S 500 \mathrm{~A}$ contendo corante $20,0 \mathrm{mg} \mathrm{L}^{-1}$, S500 B7 de palma contendo corante $18,6 \mathrm{mg} \mathrm{L}^{-1}$ e S500 B7 de palma sem corante (nenhuma das soluções foi previamente diluída)

Na Figura 3, para os óleos diesel S10 A e S500 A sem corante, a absorbância no intervalo entre 520 e $700 \mathrm{~nm}$ pode ser considerada desprezível. Quando o corante Sudan M Vermelho está presente no óleo diesel S500 A, observa-se uma banda de absorção atribuída ao corante e cujo máximo está situado em torno de $528 \mathrm{~nm}$. É também possível observar que a absorbância máxima para o corante apresenta um quase-patamar que se estende até $557 \mathrm{~nm}$, aproximadamente, ocorrendo uma queda brusca em comprimentos de onda mais altos. Para o S500 B7 de palma (sem corante) não são observadas bandas de absorção máxima nesse intervalo, situado entre $380-700 \mathrm{~nm}$, mas apenas um aumento contínuo e rápido da absorbância, principalmente em comprimentos de onda abaixo de $556 \mathrm{~nm}$. Se o corante é adicionado ao S500 B7 de palma, a absorbância desse produto é deslocada para a região cujos comprimentos de onda são atribuídos ao corante, com um ombro em torno de $557 \mathrm{~nm}$, aproximadamente. Com base nesses resultados, verifica-se a possibilidade de diferenciar os produtos nos quais é adicionado o corante Sudan M Vermelho daqueles que não o contêm.

Essa diferenciação qualitativa pode ser feita também quando o corante se encontra em concentrações relativamente baixas, conforme se observa na Figura 4. Para essa figura, foram obtidos espectros para o óleo diesel S10 B5 de palma considerando uma possível contaminação do produto por óleo diesel S500.

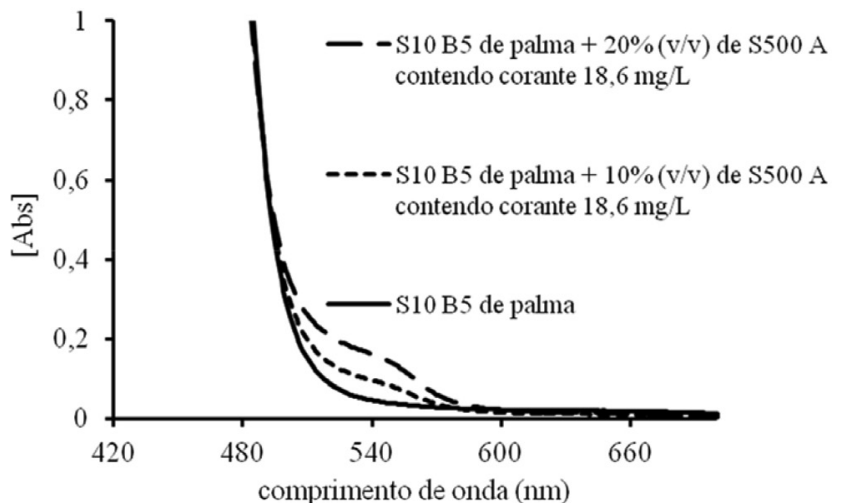

Figura 4. Espectros de absorção obtidos para o S10 B5 de palma e para misturas deste com S500 A contendo corante $18,6 \mathrm{mg} \mathrm{L}^{-1}$

\section{Quantificação do corante Sudan M Vermelho em presença da} palma

Uma vez que tenha sido possível efetuar uma diferenciação espectrofotométrica qualitativa, buscou-se avaliar a possibilidade de quantificar o corante Sudan M Vermelho em presença da palma. Para tanto, foi estabelecido o valor fixo de $570 \mathrm{~nm}$ para as medições, já que neste comprimento de onda a interferência da palma deve ser pouco significativa, como mostrado na Figura 3.

A Figura 5 mostra uma curva analítica obtida para soluções do corante Sudan M Vermelho preparadas em tolueno (que apresenta absorção desprezível no comprimento de onda de $570 \mathrm{~nm}$ ) e cujas concentrações variam entre $0,020 \mathrm{mg} \mathrm{L}^{-1} \mathrm{e} 40,0 \mathrm{mg} \mathrm{L}^{-1}$. Cada ponto corresponde à média obtida de três medições. Verifica-se, a partir desses resultados, um intervalo linear amplo, favorecendo o método para aplicações com amostras que venham conter corante dentro do intervalo especificado, que poderia ser desde o teor regulamentado pela Resolução ANP no 45/20145 (20 mg L-1) até concentrações mais baixas, oriundas de possíveis contaminações do combustível. A equação da regressão linear foi $y=0,0237 x+0,0023$, em que $y$ é a absorbância e $x$ é a concentração em $\mathrm{mg} \mathrm{L}^{-1}$. O coeficiente de determinação $\left(\mathrm{R}^{2}\right)$ foi igual a 0,9987 . Os limites de detecção $(\mathrm{LD}) \mathrm{e}$ de quantificação (LQ) apresentaram valores de $0,006 \mathrm{mg} \mathrm{L}^{-1}$ e 0,019 $\mathrm{mg} \mathrm{L}^{-1}$, respectivamente, de acordo com as equações $\mathrm{LD}=\mathrm{DPa} \times 3 / \mathrm{IC}$ e LQ $=$ DPa $\times 10 / \mathrm{IC}$ (onde DPa é o desvio-padrão do intercepto com o eixo y da curva analítica, considerada próxima ao suposto limite de quantificação, e IC é a inclinação da curva analítica). ${ }^{11}$

Os resultados para repetibilidade e precisão intermediária são resumidos na Tabela 6 . Os parâmetros foram verificados considerando o intervalo linear do método, ou seja, três concentrações (baixa, média e alta) com três replicatas cada. ${ }^{11}$

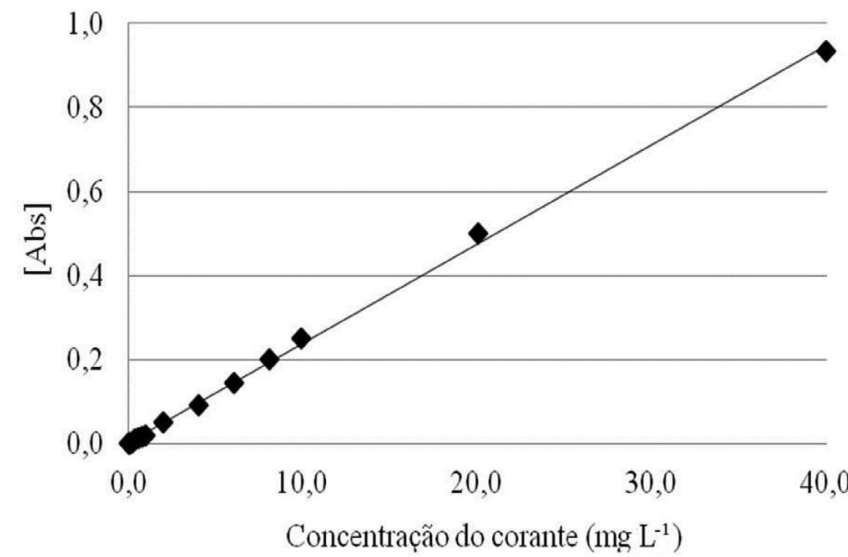

Figura 5. Curva analítica construída com padrões do corante Sudan M Vermelho em concentrações variando de $0,020 \mathrm{mg} \mathrm{L}^{-1}$ a 40,0 $\mathrm{mg} \mathrm{L}^{-1}$. Comprimento de onda $=570 \mathrm{~nm}$

Com vistas a verificar a aplicabilidade do método para a quantificação do corante Sudan M Vermelho em presença de biodiesel de palma, alguns ensaios foram conduzidos empregando diferentes amostras preparadas em laboratório (ver Tabela 3). Os resultados apresentados na Tabela 7, para as amostras, revelam diferenças relativas variando entre $2,7 \%$ e $5,2 \%$.

\section{Limitações observadas para o método de quantificação do corante Sudan M Vermelho}

A Tabela 7 ilustra apenas alguns dos resultados obtidos para a quantificação do corante Sudan M Vermelho em amostras contendo 
Tabela 6. Resultados de repetibilidade e precisão intermediária para a quantificação espectrofotométrica do corante Sudan M Vermelho. Comprimento de onda $=570 \mathrm{~nm}$

\begin{tabular}{|c|c|c|c|c|c|}
\hline Ensaio & & Amostra & $\mathrm{C}\left(\mathrm{mg} \mathrm{L}^{-1}\right)$ & $n$ & $\operatorname{DPR}(\%)$ \\
\hline \multirow{3}{*}{ Repetibilidade } & & 1 & 0,36 & 3 & 0,71 \\
\hline & & 2 & 5,61 & 3 & 0,08 \\
\hline & & 3 & 19,58 & 3 & 0,05 \\
\hline \multirow{6}{*}{ Precisão Intermediária } & \multirow{3}{*}{ Analista 1} & 1 & 0,36 & 3 & 0,71 \\
\hline & & 2 & 5,61 & 3 & 0,08 \\
\hline & & 3 & 19,58 & 3 & 0,05 \\
\hline & \multirow{3}{*}{ Analista 2} & 1 & 0,35 & 3 & 0,74 \\
\hline & & 2 & 5,53 & 3 & 0,14 \\
\hline & & 3 & 18,44 & 3 & 0,14 \\
\hline
\end{tabular}

$\mathrm{C}=$ concentração média das $n$ determinações; DPR = desvio padrão relativo.

Tabela 7. Resultados obtidos para a análise espectrofotométrica de amostras contendo corante Sudan M Vermelho em concentrações diferentes. Comprimento de onda $=570 \mathrm{~nm}$

\begin{tabular}{lccccc}
\hline Amostra & Descrição & $n$ & C média \pm DP $\left(\mathrm{mg} \mathrm{L}^{-1}\right)$ & C nominal (mg L-1) & ER (\%) \\
\hline 1 & S10 B7 de soja $+2,0 \%$ v/v de S500 B7 de palma com & 3 & $0,362 \pm 0,003$ & 0,372 & 2,7 \\
corante $18,60 \mathrm{mg} \mathrm{L}^{-1}$ & & & & \\
2 & S500 B7 de palma com corante $7,00 \mathrm{mg} \mathrm{L}^{-1}$ & 3 & $7,350 \pm 0,002$ & 7,020 & 4,7 \\
3 & S500 B7 de palma com corante $18,60 \mathrm{mg} \mathrm{L}^{-1}$ & 3 & $19,584 \pm 0,009$ & 18,612 & 5,2 \\
\hline
\end{tabular}

$\mathrm{C}=$ concentração; $n=$ número de determinações; $\mathrm{DP}=$ desvio padrão; $\mathrm{ER}=$ erro relativo.

biodiesel de óleo de palma em óleo diesel. É importante ressaltar que o óleo diesel é uma matriz extremamente complexa, apresentando compostos que podem fracamente absorver no comprimento de onda de interesse do método, dependendo de fatores relacionados à oxidação ou própria origem do combustível, por exemplo. Em concentrações muito baixas do corante $\left(<0,1 \mathrm{mg} \mathrm{L}^{-1}\right)$ estes compostos causam interferência mais significativa nas análises. Já os carotenos presentes no biodiesel de palma podem sofrer auto-oxidação em condições de armazenamento e transporte, possibilitando a formação de compostos com absortividade molar bem inferior. Pode-se considerar, também, certa imprevisibilidade nos comprimentos de onda nos quais tais compostos resultantes de oxidação podem vir a absorver. Estes fatos não invalidam o objetivo principal do trabalho, mas motivam a uma investigação mais aprofundada para futuros trabalhos, considerando tanto compostos de degradação dos carotenos quanto demais compostos presentes no óleo diesel.

\section{CONCLUSÃO}

O método apresentado como uma proposta para diferenciar amostras de óleo diesel S10 B e óleo diesel S500 B contendo biodiesel de óleo de palma se mostrou favorável no que concerne à distinção qualitativa observada nos espectros de absorção obtidos para diferentes amostras. Essa constatação, por si só, já seria suficiente para cumprir o objetivo principal do presente trabalho.

Como uma forma de complementar o estudo, avaliou-se também a possibilidade de quantificar o corante Sudan M Vermelho em presença da palma. Essa quantificação, em princípio, mostrou-se promissora, mas não completamente adequada devido a possíveis efeitos de matriz, principalmente para concentrações muito baixas do corante $\left(<0,1 \mathrm{mg} \mathrm{L}^{-1}\right)$. Dessa forma, o método de quantificação apresentado não seria aplicável para algumas situações práticas, por exemplo, nos casos em que contaminações de óleo diesel S10 B por óleo diesel S500 B viessem ser consideradas, embora a distinção qualitativa tenha se mostrado possível.

Assim, conclui-se que o projeto atingiu seu objetivo principal e, além disso, fomentou base analítica para buscar alternativas de aperfeiçoamento do método de quantificação do corante vermelho adicionado ao óleo diesel.

\section{AGRADECIMENTOS}

Às Refinarias Petrobras, REPLAN e REVAP, pelo fornecimento de amostras de óleo diesel A S10 e S500 utilizadas no presente estudo.

Às empresas Caramuru e JBS, pelo fornecimento de amostras de óleo de palma utilizadas na obtenção do biodiesel de palma.

Ao CNPq, pelo apoio financeiro (MCTI/CNPq N ${ }^{\circ} 40 / 2013$ ).

À Agência Nacional do Petróleo, Gás Natural e Biocombustíveis - ANP e à Embrapa Agroenergia, pelo apoio técnico.

\section{REFERÊNCIAS}

1. Boletim Mensal do Biodiesel. Superintendência de Refino, Processamento de Gás Natural e Produção de Biocombustíveis da Agência Nacional de do Petróleo, Gás Natural e Biocombustíveis. Disponível em http://www.anp.gov.br, acessada em Março de 2017.

2. http://www12.senado.leg.br/noticias/materias/2015/04/14/cma-aprovatexto-que-incentiva-producao-de-dende-e-libera-plantio-de-palma-naamazonia/tablet, acessada em Março de 2017.

3. http://www.biodieselbr.com/noticias/materia-prima/dende/projeto-valorizar-cadeia-produtiva-dende-080115.htm, acessada em Março de 2017.

4. Dal Prá, V.; Soares, J. F.; Monegoa, D. L.; Vendruscoloc, R.G.; Freire, D.M.G.; Alexandrie, M.; Koutinase, A.; Wagnerc, R. Mazuttib, M. A.; da Rosaa, M. B.; J. Supercrit. Fluids 2016, 112, 51.

5. Resolução ANP n ${ }^{\circ} 45 / 2014$, publicada no Diário Oficial da União de 26 de agosto de 2014. Disponível em: http://www.anp.gov.br.

6. Resolução ANP n ${ }^{\circ}$ 30/2016, publicada no Diário Oficial da União de 24 de junho de 2016. Disponível em www.anp.gov.br.

7. Corgozinho, C.N.C.; Tese de Doutorado, Universidade Federal de Minas Gerais, Brasil, 2009.

8. Remédios C. M. R.; Nunes E. C. B.; Cabral A. J. F.; del Nero J.; Alcântara Jr. P.; Moreira, S. G. C.; Resumos do Congresso Brasileiro de Engenharia e Ciências Dos Materiais, Foz do Iguaçu, Brasil, 2006. 
9. Szydłowska-Czerniak, A.; Trokowski, K.; Karlovits, G.; Szłyk, E.; Food Chem. 2011, 129, 1187.

10. Silva V. D.; Conceição J. N.; Oliveira I. P.; Filho O. P.; Conceição E.; Caires A. R. L.; $53^{\circ}$ Congresso Brasileiro Química, Rio de Janeiro, Brasil, 2013.
11. R.E. $n^{\circ} 899$ de 29 de maio de 2003 da Agência Nacional de Vigilância Sanitária - Guia para validação de métodos analíticos e bioanalíticos, publicada no Diário Oficial da União de 02 de junho de 2003. Disponível em: portal.anvisa.gov.br/. 